\title{
AN ODD THEOREM
}

\section{B. CURTIS EAVES}

Abstract. Let $C$ be a bounded convex polyhedral set and let $f: C \rightarrow C$ be continuous and piecewise linear. Using notions from complementary pivot theory, it is shown that if each fixed point of $f$ lies interior to some piece of linearity, then $f$ has an odd number of fixed points. In addition, an algorithm is given for computing a fixed point of $f$.

1. Introduction. Using the main ideas of complementary pivot theory (see [1]-[10]), we prove the following theorem.

THEOREM. Let $C$ be a bounded convex polyhedral set and let $f: C \rightarrow C$ be continuous and piecewise linear. If each fixed point of $f$ lies interior to some piece of linearity, then $f$ has an odd number of fixed points.

An algorithm for computing a fixed point of $f$ (whether or not the interior condition is met) is a by-product of the proof of the theorem.

The essential difference between our atttitude and that of [8]-[11] and hence Sperner's Lemma is that we label vertices of a triangulation with real vectors instead of integers or integer vectors and that we define a simplex to be "completely labeled" if a convex combination of the vector labels generate zero. Our development continues the study of vector labeled pseudomanifold-like structures as in [1]-[8]. This paper closely parallels the last section of Scarf [8]; however, our choice of "pseudomanifold" evidently has certain advantages.

2. Graph principle. Our proof will rest on a simple graph principle; the same principle used in [1]-[10]. By a graph $(N, A)$, we mean a finite set $N$ together with a symmetric antireflexive relation $A$ on $N$. If $a A b$ (hence $b A a$ and $a \neq b$ ), we say $a$ and $b$ are adjacent. We call an element $a$ of $N$ odd or even if it is adjacent to an odd or even number of elements of $N$, respectively. Recall that a graph has an even number of odd elements. In the next section, we construct a particular graph and use this device to prove our theorem. In this graph each element will be adjacent to exactly one or two elements; in this case, the odd elements have a natural pairing.

3. The theorem and proof. Let $C$ be a finite dimensional bounded convex polyhedral set. We can assume that $C$ lies in $n$-dimensional

Received by the editors July 17, 1969.

AMS 1968 subject classifications. Primary 5485, 0504.

Key words and phrases. Odd number, fixed points, piecewise linear, simplicial, complementary pivots, Sperner's Lemma, pseudomanifold. 
Euclidian space and that it has an interior. Let $T$ be a triangulation of $C$ (i.e., $T$ is a complex and $|T|=C$, see [12]), and let $f: C \rightarrow C$ be a continuous function which is linear (i.e., affine) on each simplex of $T$. Let $(C, T, f)$ denote such a triple. If each fixed point of $f$ is interior to an $n$-simplex of $T$, then we say that $(C, T, f)$ is nondegenerate. To prove our result, it is sufficient to prove the following theorem.

ThEOREM. If $(C, T, f)$ is nondegenerate, then $f$ has an odd number of fixed points.

Given $(C, T, f)$ we notice that $f$ is completely determined by its action on the vertices of $T$. Indeed, if $r \in S \in T$, then $f(r)=\sum_{s} f(s) x_{s}$ where $r=\sum_{s} s x_{s}, \sum_{s} x_{s}=1$, and $x_{8} \geqq 0$ (where $s$ ranges over the vertices of $S$ ).

A simplex $S$ of $T$ contains a fixed point if and only if the system

$$
\sum_{s}(f(s)-s) x_{s}=0, \quad \sum x_{s}=1
$$

has a nonnegative solution in the $x_{s}$ (where $s$ ranges over the vertices of $S)$. In this case $\sum_{\mathrm{s}} s x_{\mathrm{s}}$ is the fixed point. If $(C, T, f)$ is nondegenerate, then it follows that a simplex will contain at most one fixed point; if $S$ contains a fixed point, then $S$ is an $n$-simplex and the solution $x_{8}$ of the system above is unique and positive.

Assume $(C, T, f)$ is nondegenerate. Let $C^{\prime}$ be an $n$-simplex which contains $C$ in its interior. We shall extend both $T$ and $f$ to $C^{\prime}$ to generate $\left(C^{\prime}, T^{\prime}, f^{\prime}\right)$. Let $v_{0}, \cdots, v_{n}$ be the vertices of $C^{\prime}$.

Extend $T$ to a triangulation $T^{\prime}$ of $C^{\prime}$ without introducing new vertices; that is, a vertex of $T^{\prime}$ is either a vertex of $T$ or a vertex of $C^{\prime}$. Hence each $(n-1)$-face of $C^{\prime}$ is an element of $T^{\prime}$.

Temporarily let $r$ be any point of $C$. Define $f^{\prime}$ on $C^{\prime}$ by setting $f^{\prime}(t)=f(t)$ for vertices of $T$ and $f^{\prime}\left(v_{i}\right)=r$ for vertices of $C^{\prime}$ and then by extending $f^{\prime}$ linearly on the simplexes of $T^{\prime}$. Now we further specify $r$. Select $r \in C$ such that for any $(n-1)$-simplex $S$ of $T^{\prime}$ the system

$$
\left(f^{\prime}\left(v_{0}\right)-v_{0}\right) x_{S}+\sum_{s}\left(f^{\prime}(s)-s\right) x_{s}=0, \quad x_{S}+\sum_{s} x_{s}=1
$$

either has a unique positive solution in $x_{S}$ and the $x_{8}$ or else has no nonnegative solution (where $s$ ranges over the vertices of $S$ ). Such $r$ 's are very available; in fact, almost every element of $C$ will suffice.

We can now define a particular graph. Let $\left(C^{\prime}, T^{\prime}, f^{\prime}\right)$ be generated as just described. Let $N_{1}$ be the set of simplexes of $T^{\prime}$ which contain fixed points; these simplexes will be $n$-simplexes of $T$. Let $N_{2}$ be the set of $(n-1)$-simplexes $S$ in $T^{\prime}$ for which the system 
1970]

$$
\left(f^{\prime}\left(v_{0}\right)-v_{0}\right) x_{S}+\sum_{s}\left(f^{\prime}(s)-s\right) x_{s}=0, \quad x_{S}+\sum_{s} x_{s}=1
$$

has a nonnegative solution in $x_{S}$ and the $x_{8}$ (where $s$ ranges over the vertices of $S$ ); these solutions will be positive and unique. Let $N$ $=N_{1} \cup N_{2}$. We define two distinct simplexes of $N$ to be adjacent if they lie in a common simplex of $T^{\prime}$.

Let $S_{0}$ be the $(n-1)$-simplex with vertices $\left\{v_{1}, \cdots, v_{n}\right\}$. One can now establish that $S_{0} \in N_{2}$, that each element of $N_{1} \cup\left\{S_{0}\right\}$ is adjacent to exactly one element of $N$, and that each element of $N_{2} \sim\left\{S_{0}\right\}$ is adjacent to exactly two elements of $N$. From the graph principle, we see that $N_{1}$ contains an odd number of elements; this establishes the theorem.

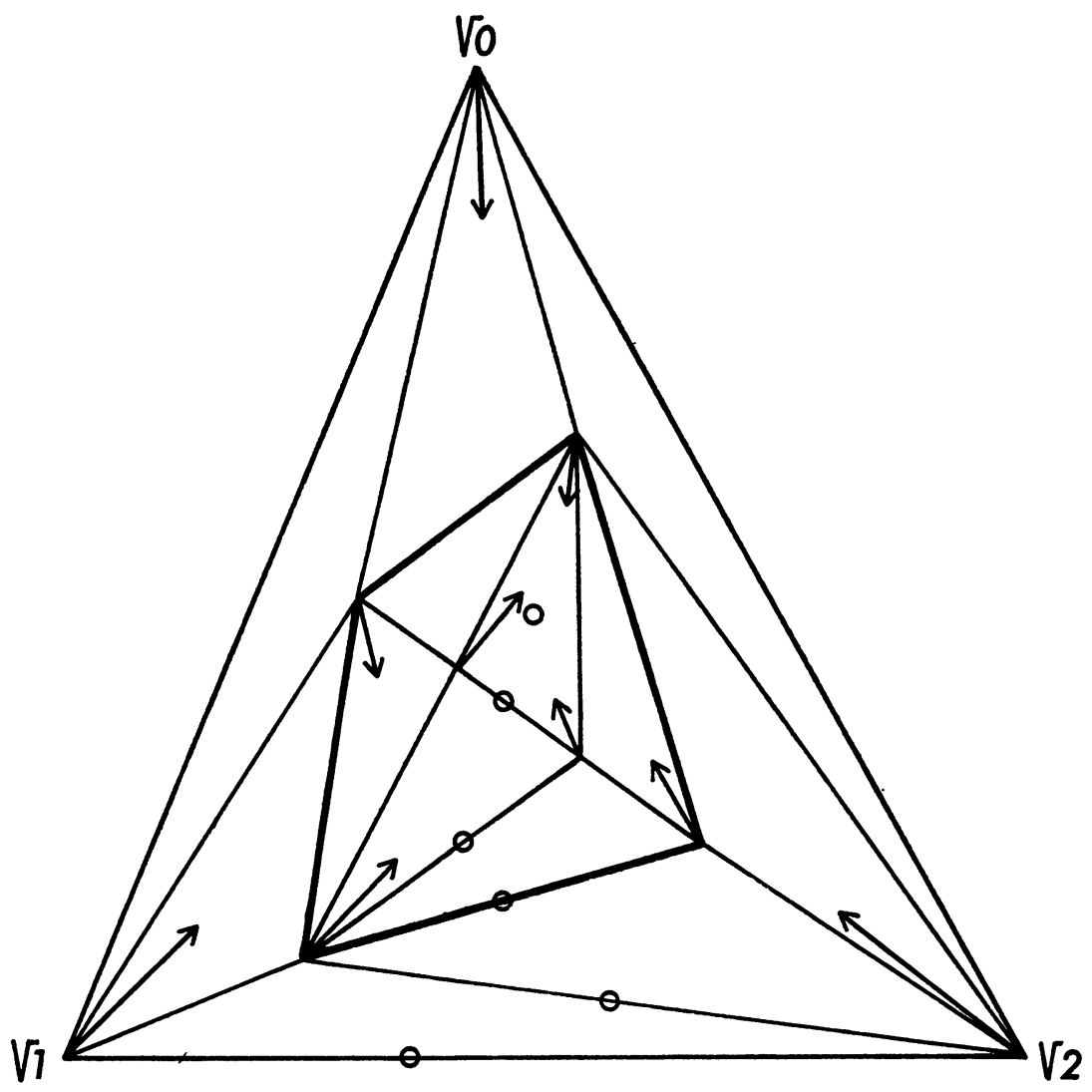

The figure illustrates the structure for a 2-dimensional $C$. The arrow at a vertex $t$ denotes the direction of $f^{\prime}(t)-t$ (further specifica- 
tion is unnecessary), the heavy lines denote the boundary of $C$, and the small circles denote the simplexes which are in $N$.

4. The algorithm. The preceding development gives a procedure for calculating a fixed point of $(C, T, f)$. After constructing $\left(C^{\prime}, T^{\prime}, f^{\prime}\right)$, one begins at $S_{0}$ and proceeds to an adjacent simplex, etc. This step from simplex to simplex is essentially a "pivot" as known in linear programming. One eventually terminates with a simplex containing a fixed point, and hence, one has the fixed point.

The next section shows that if $(C, T, f)$ is degenerate, the algorithm may still be applied to find a fixed point $((C, T, f)$ is altered slightly to make it nondegenerate; however, from solely computational considerations, there are far more efficient methods of dealing with degeneracy).

The section on Brouwer's theorem demonstrates that if $g: C \rightarrow C$ is a continuous function and if $\epsilon>0$, then the algorithm can be used to compute a point $t \in C$ such that $|g(t)-t| \leqq \epsilon$. Scarf's procedure [8] has this capability.

Kuhn [4] describes an extremely efficient data handling procedure which can be adapted to our algorithm.

5. Perturbation and stability. Here we show that nondegeneracy is stable and that nondegeneracy can be achieved via a perturbation.

Suppose that $(C, T, f)$ is nondegenerate. Then there is an $\epsilon>0$ such that $(S, T, g)$ is nondegenerate and such that a simplex of $T$ will contain a fixed point of $f$ if and only if it contains a fixed point of $g$, if $|f-g| \leqq \epsilon$.

Consider $(C, T, f)$ and $(C, T, g)$. Suppose that $g(C)=r$ and that $r$ is interior to an $n$-simplex of $T$. Then there is an $\epsilon_{0}>0$ such that for $0<\epsilon \leqq \epsilon_{0},(C, T,(1-\epsilon) f+\epsilon g)$ is nondegenerate. Further, if a simplex of $T$ contains a fixed point of $(1-\epsilon) f+\epsilon g$, then it contains a fixed point of $f$ for $0<\epsilon \leqq \epsilon_{0}$.

6. Brouwer's theorem and extensions. From $\S \S 4$ and 5 we see that for any $(C, T, f)$ there is a fixed point. We can now prove Brouwer's fixed point theorem.

Let $g: C \rightarrow C$ be a continuous function. Choose $\left(C, T_{n}, f_{n}\right)$ such that $\left|f_{n}-g\right| \leqq 1 / n$ for $n=1,2, \ldots$. Let $s_{n}$ be a fixed point of $f_{n}$. We have $\left|g\left(s_{n}\right)-s_{n}\right| \leqq 1 / n$. If $s$ is a cluster point of the $s_{n}$ sequence, then clearly $s$ is a fixed point of $g$.

Professor Edwin Spanier has shown me a different proof of the theorem of this paper which involves summing the degrees of the fixed points. The results obtained were slightly stronger. 


\section{REFERENCES}

1. R. W. Cottle, and G. B. Dantzig, Complementary pivot theory of mathematical programming, Linear Algebra and Appl. 1 (1968), no. 1, 103-125. MR 37 \#2515.

2. B. Curtis Eaves, Linear complementarity problem in mathematical programming, Doctoral Dissertation, Stanford University, Stanford, Calif., 1969.

3. C. E. Lemke, Bimatrix equilibrium points and mathematical programming, Management Sci. 11 (1964/65), 681-689. MR 32 \#7243.

4. C. E. Lemke, and J. T. Howson, Jr., Equilibrium points of bimatrix games, J. Soc. Indust. Appl. Math. 12 (1964), 413-423. MR 30 \#3769.

5. K. G. Murty, On the number of solutions to the complementary quadratic programming problem. Doctoral Dissertation, Engineering Science, University of California, Berkeley, Calif., 1968.

6. T. Hansen, On the approximation of a competitive equilibrium, Ph.D. Dissertation, Yale University, New Haven, Conn., 1968.

7. H. Scarf, The core of an $N$ person game, Econometrica 35 (1967), 50-69. MR 38 \#3051.

8. - The approximation of fixed points of a continuous mapping, SIAM J. Appl. Math. 15 (1967), 1328-1343. MR 39 \#3814.

9. D. I. A. Cohen, On the Sperner lemma, J. Combinatorial Theory 2 (1967), 585587. MR 35 \#899.

10. Harold W. Kuhn, Simplicial approximation of fixed points, Proc. Nat. Acad. Sci. U.S.A. 61 (1968), 1238-1242.

11. - Some combinatorial lemmas in topology, IBM J. Res. Develop. 4 (1960), 508-524. MR 23 \#A1358.

12. L. S. Pontryagin, Foundations of combinatorial topology, OGIZ, Moscow, 1947; English transl., Graylock Press, Albany, N. Y., 1952. MR 11, 450; MR 14, 194.

Stanford University, Stanford, California 94305 